\title{
Richard B. Gunderman: Essential Radiology: Clinical Presentation, Pathophysiology, Imaging (3rd edn.)
}

\author{
Thieme Medical Publishers, New York, 2014, ISBN: 978-1-60406-573-2
}

\author{
Davide D'Arienzo • Luigi Mansi
}

Published online: 30 July 2014

(C) Springer-Verlag Berlin Heidelberg 2014

This is the third edition of a book edited by Richard B. Gunderman, Vice Chairman of the Department of Radiology and Professor of Radiology at the Indiana University of Indianapolis. The volume is organized into ten chapters for a total of 325 pages, including a large number of high-quality figures, tables and illustrations and a brief appendix on normal crosssectional anatomy.

The first chapter, "Introduction to Radiology", after a brief history of the origins of this medical specialty from the insights of Wilhelm Roentgen, well describes the basic concepts and the physical principles of the most important techniques constituting diagnostic imaging, including transmission imaging (traditional radiology and computed tomography), reflection imaging (ultrasound), emission imaging (magnetic resonance and nuclear medicine). In this chapter are also described contrast agents, angiography, interventional radiology and principles of medical imaging. The second chapter, entitled "The Circulation System: The Heart and The Great Vessels", illustrates the role of each imaging modality in the evaluation of the heart and great vessels. Cardiovascular imaging strategies applied in the most common pathological conditions, starting from the understanding of their clinical presentation and of the underlying pathophysiology, are clearly presented. The third chapter, "The Respiratory System", describes the approach of medical imaging to pathologies involving the respiratory system, focusing on technical features, clinical presentation and typical patterns of pulmonary and airways abnormalities. The fourth and fifth chapters entitled, respectively, "The Digestive System" and "The Urinary Tract". The basic pathophysiology, clinical presentation and imaging strategies to apply in the most relevant diseases involving the two systems are clearly explained. In the sixth chapter, "The Acute Abdomen", the author focuses on the critical radiological signs of the most common conditions that lead to acute abdominal pain, providing a general imaging approach to the problem. The role of nuclear medicine in the diagnosis and of interventional radiology in the treatment of acute diseases are also discussed in this chapter.. The following three chapters are "The Reproductive System", "The Musculoskeletal System" and "Neuroimaging". The last chapter, "Pediatric Radiology", describes the most common paediatric medical and surgical pathologies, reviewing some of the main anatomical and pathophysiological differences between children and adults.

The goal of the book is well expressed in the title, "Essential Radiology". It clearly defines the range of information and images available concerning radiological techniques. In this sense, the persistent interest in traditional X-ray procedures is noteworthy. These procedures continue to represent an important element in medical education and training. "Essential" also means that the main focus of the book is elementary information usable in standard routine practice. The negative counterpart to this approach is the limited analysis and evaluation of radionuclide procedures, even though the primary role today is occupied by PET and more generally techniques utilizing hybrid machines. For these reasons, this publication can be considered a textbook mainly directed to medical students, that may also be useful in the library of a nuclear medicine department as a fast reference book where it is possible to find easily and quickly helpful information to utilize in routine clinical practice.

D. D’Arienzo $\cdot$ L. Mansi $(\square)$

Second University of Naples, Naples, Italy

e-mail: luigi.mansi@unina2.it 